\title{
Spatial Learning Impairment Parallels the Magnitude of Dorsal Hippocampal Lesions, but Is Hardly Present following Ventral Lesions
}

\author{
Edvard Moser, May-Britt Moser, and Per Andersen \\ Department of Neurophysiology, Institute of Basic Medical Sciences, University of Oslo, 0317 Oslo, Norway
}

\begin{abstract}
The hippocampus plays an essential role in spatial learning. To investigate whether the whole structure is equally important, we compared the effects of variously sized and localized hippocampal aspiration lesions on spatial learning in a Morris water maze. The volume of all hippocampal lesions was determined. Dorsal hippocampal lesions consistently impaired spatial learning more than equally large ventral lesions. The dorsal lesions had to be larger than $20 \%$ of the total hippocampal volume to prolong final escape latencies. The acquisition rate and precision on a probe test without platform were sensitive to even smaller dorsal lesions. The degree of impairment correlated with the lesion volume. In contrast, the lesions of the ventral half of the hippocampus spared both the rate and the precision of learning unless nearly all of the ventral half was removed. There was no significant effect of the location (dorsal or ventral) of damage to the overlying neocortex only. In conclusion, the dorsal half of the hippocampus appears more important for spatial learning than the ventral half. The spatial learning ability seems related to the amount of damaged dorsal hippocampal tissue, with a threshold at about $20 \%$ of the total hippocampal volume, under which normal learning can occur.
\end{abstract}

[Key words: hippocampus, spatial learning, memory, dorsal hippocampus, ventral hippocampus, water maze, CA1, lesion volume]

There is much evidence indicating that the hippocampus is critically involved in spatial learning and memory (see Amaral and Witter, 1991). First, some pyramidal cells in the rat hippocampus discharge rather selectively at specific locations of a spatial environment (O'Keefe and Dostrovsky, 1971; Muller et al., 1987) and maintain their receptive field when the relevant spatial cues are removed (O'Keefe and Conway, 1978) or when the light is turned off (Quirk et al., 1990), suggesting that some hippocampal pyramidal cells may subserve the memory for spatial information. Second, lesions of the hippocampus result in impaired acquisition of tasks that depend on spatial strategies

\footnotetext{
Reccived Oct. 28, 1992; revised Mar. 19, 1993; accepted Mar. 23, 1993.

This research was supported by the Norwegian Medical Research Council (NAVE/ RMF), Grants 326.91-024 and 326.88-007. We gratefully acknowledge the technical assistance of Bruce Piercey, Trond Reppen, Tore Eriksen, Eva Aaboen IFansen, and Luc Scholte.

Correspondence should be addressed to Per Andersen, Department of Neurophysiology, Institute of Basic Medical Sciences, University of Oslo, Post Box 1104 Blindern, 0317 Oslo, Norway.

Copyright (C) 1993 Society for Neuroscience $0270-6474 / 93 / 133916-10 \$ 05.00 / 0$
}

for their solution, such as the Morris water maze (Morris et al. 1982, 1990; Sutherland et al., 1983) and the radial-arm maze (Jarrard, 1978; Olton et al., 1978). No impairment is observed in such tasks when the target can be located with nonspatial cues. However, accumulating evidence suggests that hippocampal lesions impair several forms of nonspatial learning as well, for example, odor discrimination (Eichenbaum et al., 1988, 1989) and configural discrimination (Rudy and Sutherland, 1989; Suthcrland et al., 1989).

It is not known whether the learning requires the entire hippocampus. The lamellar organization of the intrahippocampal pathways (Andersen et al., 1971) argues for a unitary mode of operation along the entire septotemporal extent of the hippocampus. Learning, accordingly, might be subserved by any part of the septotemporal axis of the hippocampus, although cooperation between neighboring areas could be required, possibly through longitudinal connections (Amaral and Witter, 1989). On the other hand, external connections suggest a functional division between the dorsal and ventral parts of the structure. The dorsal part of the hippocampus receives fibers from a lateral strip of the entorhinal cortex, while the ventral hippocampus is served by more medial cells (rat: Ruth et al., 1982; cat: Witter and Groenewegen, 1984; monkey: Witter et al., 1989). Sensory information from various visual, auditory, and somatosensory association areas and from the olfactory bulb reaches predominantly the dorsal half of the hippocampus by way of the lateral entorhinal area, directly or relayed through the perirhinal cortex (Jones and Powell, 1970; van Hoesen and Pandya, 1975; Deacon et al., 1983; Room and Groenewegen, 1986; Insausti et al., 1987). Thus, the dorsal hippocampus seems better served than the ventral hippocampus by sensory signals needed for a spatial learning task.

The functional significance of the changing connectivity of cells along the septotemporal axis of the hippocampus is largely unknown. Early work suggested impairment on a wide range of learning tasks following both dorsal and ventral hippocampal lesions (Nadel, 1968; Stevens and Cowey, 1973). Usually, different aspects of performance were affected by the two lesions. Some studies reported larger maze learning impairments following dorsal than ventral lesions of the hippocampus (Hughes, 1965; Sinnamon et al., 1978), but others found no differences (Gross et al., 1965). However, none of these studies controlled for differences in the size of the dorsal and ventral lesions. Therefore, we have made dorsal and ventral hippocampal lesions of various sizes, estimated the volume of the hippocampal tissue that had been damaged, and measured spatial learning in a Morris water maze (Morris, 1984). 


\section{DORSAL}

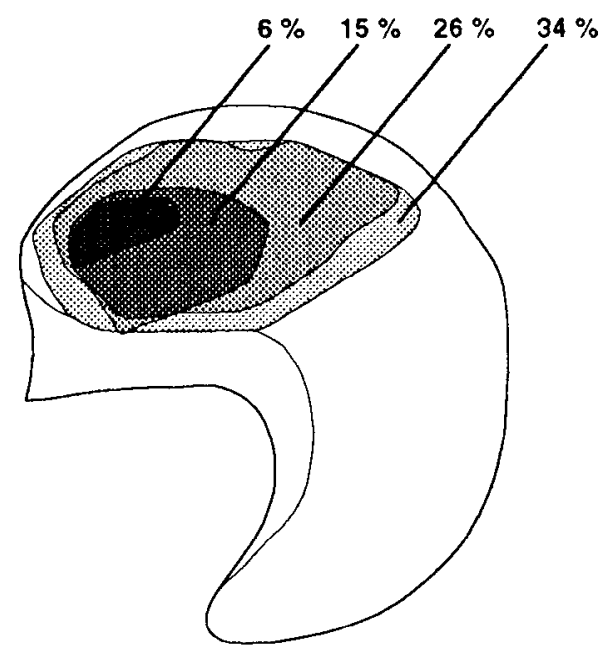

\section{VENTRAL}

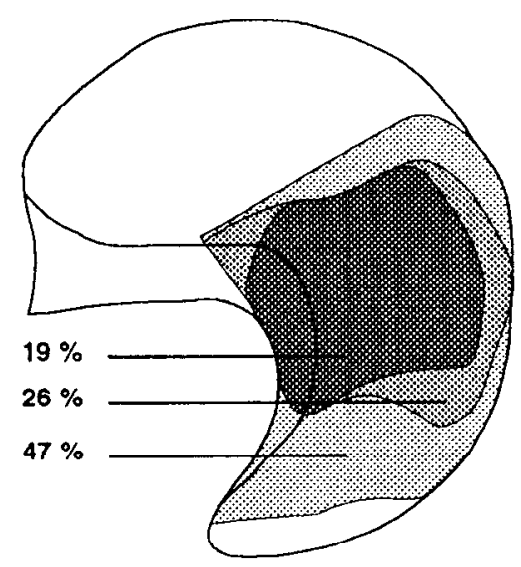

Figure 1. Reconstruction of the hippocampal lesion of seven rats with various degrees of damage to the dorsal or the ventral hippocampus. These tracings with different shadings give the median value of lesions in each of four dorsal lesion groups (DORSAL;0-10\%, $10-20 \%, 20-30 \%$, and $30-37 \%$ ) and three ventral lesion groups (VENTRAL; $15-22 \%, 23-30 \%$, and $39-52 \%)$.

\section{Materials and Methods}

Subjects and surgical procedure. Experimentally naive female LongFvans rats (180-250 gm), purchased from Möllegaards Avlslaboratorium (Denmark), were housed in groups of five to eight in transparent polycarbonate cages $(59 \times 38 \times 20 \mathrm{~cm})$ with food and water available ad libitum. They were kept on a $12 \mathrm{hr}: 12 \mathrm{hr}$ light/dark schedule and tested in the light phase.

The rats were randomly assigned to the following treatment groups: dorsal hippocampal lesions, ventral hippocampal lesions, dorsal neocortical control lesions, ventral neocortical control lesions, and controls with dorsal or ventral sham operations (the dura was not cut). The animals were anesthetized with a mixture (Equithesin) of $153 \mathrm{mg}$ of chloral hydrate, $35 \mathrm{mg}$ of pentobarbital, and $75 \mathrm{mg}$ of $\mathrm{MgSO}_{4}$ per kilogram of body weight, and placed in a stereotaxic frame. The skull was exposed, the temporal muscles retracted (ventral groups), and holes drilled in the skull on its dorsal (dorsal groups) or temporal sides (ventral groups). Using an operating microscope, we first aspirated some of the occipitoparietal (dorsal hippocampal and dorsal neocortical groups) or occipitotemporal (ventral hippocampal and ventral neocortical groups) cortex, including the corpus callosum, and then, in the dorsal and ventral hippocampal groups, part of the underlying hippocampus. We always tried to leave the fimbria intact (dorsal hippocampal groups). The size of the hippocampal lesions, particularly their septotemporal extent, was varied. All lesions were bilateral and were intended to be symmetrical.

Behavioral testing. Behavioral testing was conducted in a Morris water maze (Morris, 1984), a circular polyvinylchloride tank $(198 \mathrm{~cm}$ diameter, $50 \mathrm{~cm}$ deep) with a featureless, white inner surface. The pool was filled to a depth of $40 \mathrm{~cm}$ with $23 \pm 2^{\circ} \mathrm{C}$ water to which 3 liters of milk were added. A translucent cylindrical Plexiglas platform $(11 \mathrm{~cm}$ diameter) was submerged $1.5 \mathrm{~cm}$ below the water surface at one out of four places, $90^{\circ}$ apart, midway between the center and periphery of the pool. In control trials, the platform was raised $3 \mathrm{~cm}$ and a stripe of colored tape was fastened around its upper perimeter. The maze was located in a well-lit room $(3 \times 4 \mathrm{~m})$ with numerous visual cues. $\Lambda$ computer in an adjacent room, connected to a vertically oriented video camera above the water maze, identified the black head of the swimming rat and stored its position at $10 \mathrm{~Hz}$. The data could be retrieved for later analysis.

Training the rats to locate the platform in the water maze started 1 week after surgery. Each rat was assigned a platform position that was maintained throughout the experiment. The rats were trained twice daily. The two sessions, which both consisted of four trials $40 \mathrm{sec}$ apart, were separated by at least $4 \mathrm{hr}$. Session 13 was preceded by a $60 \mathrm{sec}$ spatial probe test on which the platform was removed. On sessions 1415 , the platform was visible. On session 16 , it was submerged again.

Each trial was begun by releasing the rat into the water, with its face toward the pool wall, at one out of 12 randomly varied and equally spaced start positions. Vision was blocked until the go-signal. The program tracked the position of the rat until the rat entered the platform. If the rat failed to find the platform within $120 \mathrm{sec}$, it was guided onto it. The rat was always left on the platform for $30 \mathrm{sec}$. On the spatial probe test, the rats were released from the quadrant opposite to the previous platform quadrant and the swimming was tracked for $60 \mathrm{sec}$. Between and after trials, the rats rested under a heating lamp in a separate cage.

Histology. Within a day after finishing the behavioral testing, the rats were killed with an overdose of Equithesin and perfused intracardially with saline and $4 \%$ formaldehyde. The brains were removed and stored in formaldehyde. Frozen sections $(20 \mu \mathrm{m})$ were cut coronally and every tenth section stained with cresyl violet. Outlines of the relevant hippocampal tissue characteristics and lesions were traced onto line drawings of 16 coronal sections covering the entire hippocampus (taken from König and Klippel, 1963). The outlines were then digitized. Morphological criteria (cell layer features) were used to determine the borders between the CAI on one hand and the subiculum and the C.A3 region on the other. The volume of the lesions in the subiculum, the hippocampus with the dentate gyrus, the CA1 subfield, and the neocortex were then estimated separately. Neocortical and hippocampal lesion volumes were calculated by treating the lesions as a series of truncated cones with parallel surfaces equal to the areas inside the lesion outlines. The hippocampal lesion volumes were expressed as percentage of the normal total volume of the two hippocampi.

Statistical procedures. The results were evaluated with mixed-model (split-plot) repeated-measures analysis of variance. To reduce the heterogeneity of variance, the latency data were log-transformed prior to the analysis. To correct for violations of sphericity (Vasey and Thayer, 1987), we multiplied the numerator and denominator degrees of freedom of the $F$ ratio by Huynh and Feldt (1976) estimate of $\epsilon$ (these products are the $\mathrm{df}$ reported).

\section{Results}

\section{Description of the lesions}

Dorsal hippocampal lesions. In all rats with acceptable dorsal hippocampal lesions, the damage was confined to the dorsal half of the hippocampus bilaterally (Figs. 1, 2). Ischemia-induced necrosis was seen only in the immediate vicinity of the aspiration borders. No damage of the fimbria could be seen by eye. The estimated amount of hippocampal damage in the dorsal hippocampal groups ranged from $1.9 \%$ to $37.0 \%$ of the total hippocampal tissue. The distribution of the lesion volumes is shown in Figure 5. The largest lesions included nearly all tissue between the anterior tip of the hippocampus and a horizontal 
DORSAL $6 \%$

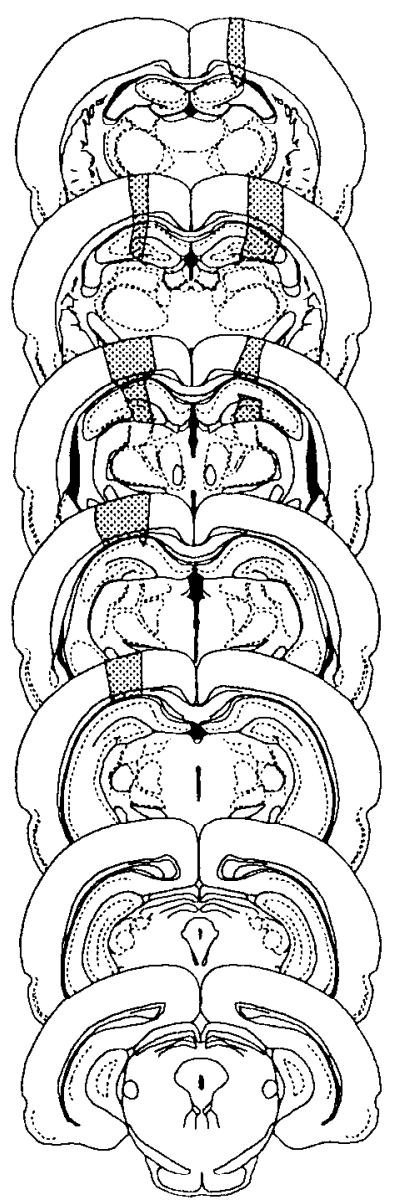

DORSAL $15 \%$

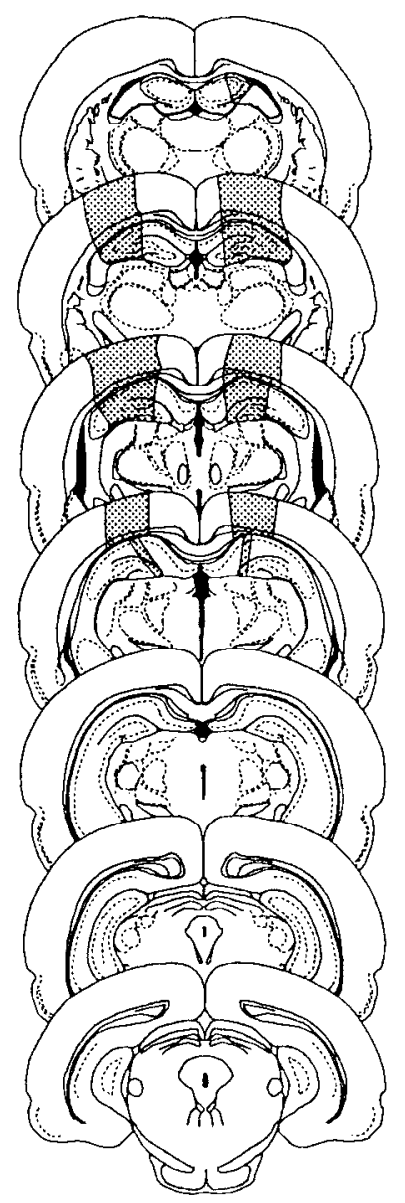

DORSAL $26 \%$

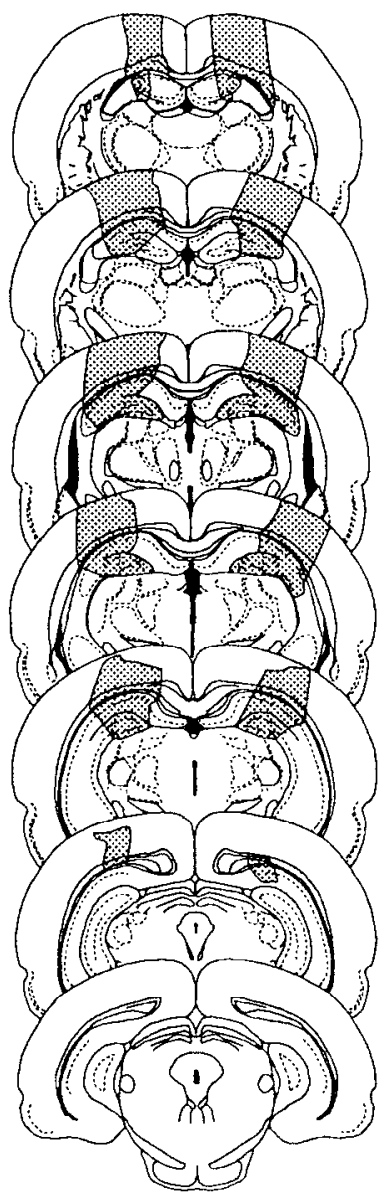

DORSAL $34 \%$

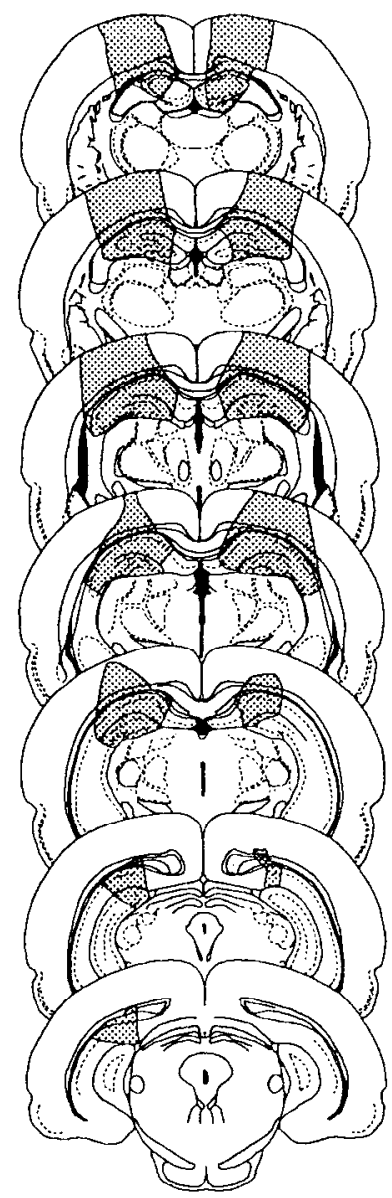

Figure 2. Dorsal hippocampal lesions: reconstruction of the lesions in four rats who had damage to $6 \%, 15 \%, 24 \%$, and $34 \%$ of the total hippocampal tissue. These examples are the median lesion volumes in each of the dorsal groups, which comprised $0-10 \%, 10-20 \%, 20-30 \%$, and $30-37 \%$ damage, relative to the total hippocampal volume.

plane through the dorsal part of the LGN (Fig. 2). In rats with smaller lesions (10-20\%), the lesions terminated more septally. The medial parts $(1-2 \mathrm{~mm})$ of the dentate gyrus and the CAl were spared at the septal end of the hippocampus, whereas the $\mathrm{CA} 3$ and the adjoining part of CA1 were spared toward the caudal end of the lesions. The smallest dorsal hippocampal lesions $(<10 \%)$ consisted of a hole through the CAl, usually also affecting a part of the CA3 and the dentate gyrus, and were placed in the septal one-quarter of the hippocampus.

Ventral hippocampal lesions. The ventral hippocampal lesions were restricted to the temporal three-fifths of the scptotemporal axis bilaterally (Figs. 1, 3). Estimates of the damage ranged from $16.0 \%$ to $52.6 \%$ of the total hippocampal tissue volume. The fimbria was partly or completely transected in all rats with ventral hippocampal lesions. The most extensive lesions included nearly all tissue between the temporal pole of the hippocampus and a horizontal plane through the dorsal part of the LGN. The main difference between large and small ventral hippocampal lesions was the amount of tissue spared at the ventral tip of the hippocampus and in the most caudal part of the dentate gyrus and the CAl.

Neocortical damage. Rats with acceptable control lesions had no or negligible hippocampal damage, but the overlying corpus callosum was partially severed. In both the dorsal hippocampal and dorsal neocortical groups, the neocortical damage included parts of the hindlimb area of the somatosensory cortex, occipital cortical area 2 and parietal cortical area 1 (Zilles, 1985). In the ventral groups, the temporal cortex (areas 1-3) and small parts of occipital cortical area 2 and posterior parietal cortical area 1 were affected. The estimated amount of neocortical damage was comparable in the groups with hippocampal lesions and their respective control groups: $20.6 \pm 1.0$ and $23.3 \pm 2.3 \mathrm{~mm}^{3}$ (mean \pm SEM) in the dorsal hippocampal and dorsal neocortical groups, respectively, and $38.2 \pm 1.8$ and $34.1 \pm 3.8 \mathrm{~mm}^{3}$ in the ventral hippocampal and ventral ncocortical groups.

Unintended damage. In the dorsal and ventral hippocampal groups, damage to the subiculum was estimated to $5.5 \pm 0.8 \%$ and $25.4 \pm 4.6 \%$ of the subicular volume, respectively. However, the subicular damage was pronounced only in the rats with the largest hippocampal lesions (>30\%): $12.7 \pm 1.6 \%$ of the subiculum in the dorsal hippocampal group, and $41.3 \pm 2.5 \%$ in the ventral hippocampal group. In the thalamus, superficial lesions in the lateral posterior nucleus were seen in four rats with dorsal hippocampal lesions. In addition, small patches of gliosis were often observed beneath apparently healthy tissue in the lateral and lateral posterior nuclei. Two ventral hippocampal animals had slight damage to the ventral part of the lateral geniculate nucleus, and six ventral hippocampal lesions 

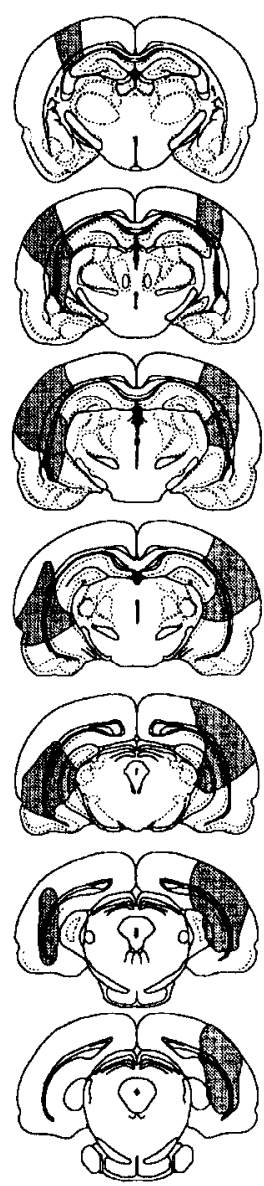

VENTRAL $19 \%$
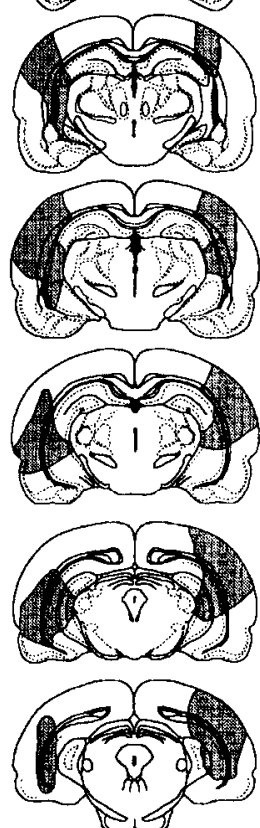

VENTRAL $26 \%$
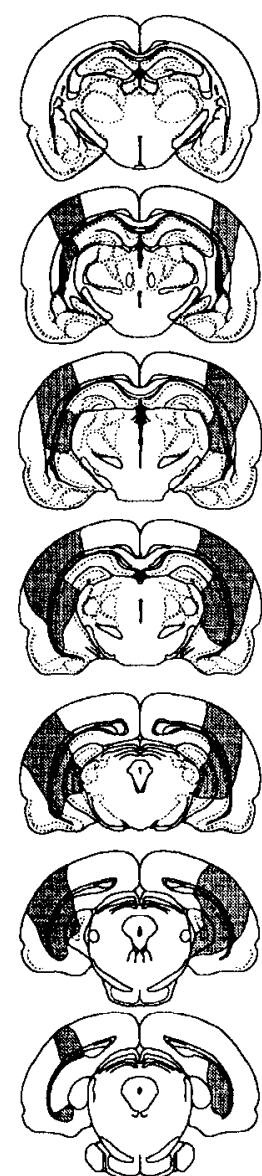

Figure 3. Ventral hippocampal lesions: reconstruction of the lesions in three rats who had damage to $19 \%, 26 \%$, and $47 \%$ of the total hippocampal tissue. These examples are the median lesion volumes in each of the ventral groups, which comprised $15-22 \%, 23-30 \%$, and $39-52 \%$ damage, relative to the total hippocampal volume.
VENTRAL $47 \%$
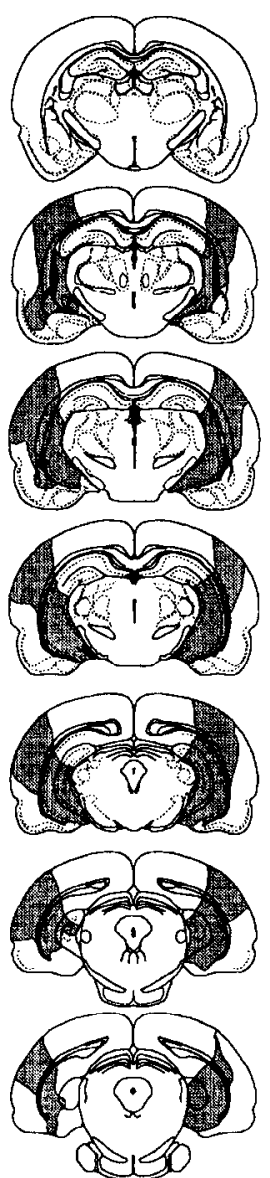

extended into the caudal parts of the amygdala. Damage to the entorhinal cortex was hard to evaluate because the brains were sectioned coronally, but a partial damage may have occurred in some rats in the ventral hippocampal group. Finally, four dorsal and five ventral neocortical rats had tiny $\left(<0.1 \mathrm{~mm}^{3}\right)$ unilateral or bilateral (two rats in the dorsal group) cuts in the stratum oriens of CAl.

Clear damage to the $L G N$ was the reason for exclusion of seven animals. Other animals were discarded due to unwanted extensive lesions in the anterior thalamus (one rat in the dorsal hippocampal group), the entorhinal cortex (one rat in the ventral hippocampal group), the fimbria (one rat in the dorsal hippocampal group), or the CA1 (three rats in the dorsal neocortical group). After these exclusions, there were 47 rats in the dorsal hippocampal, 17 rats in the ventral hippocampal, 11 rats in the dorsal neocortical, and 9 rats in the ventral neocortical group.

\section{Behavior}

With time, the animals in all groups learned to find the hidden platform progressively faster (Fig. 4). However, whereas the control groups and all rats with ventral lesions, affecting less than approximately $45 \%$ of the hippocampal tissue, learned quickly and finally swam directly to the hidden platform, the rats with dorsal lesions generally failed to learn the exact position of the target. This was expressed in longer latency to find the platform (Fig. 4) and poor performance on the spatial probe test (Fig. 5).

\section{Acquisition}

Only 12 of 84 rats, distributed evenly across the groups, found the platform on the first trial of the first session. The subsequent behavior depended heavily on the location and the size of the lesion (Fig. 4). Rats with dorsal damage amounting to less than $20 \%$ of the total hippocampal tissue volume learned to find the submerged platform as efficiently as the neocortical control animals ( $<12 \mathrm{sec}$ at the end of the training period). However, the rate of acquisition was retarded in animals in which $10-20 \%$ was damaged. Dorsal lesions, measuring more than $20 \%$ of the
A

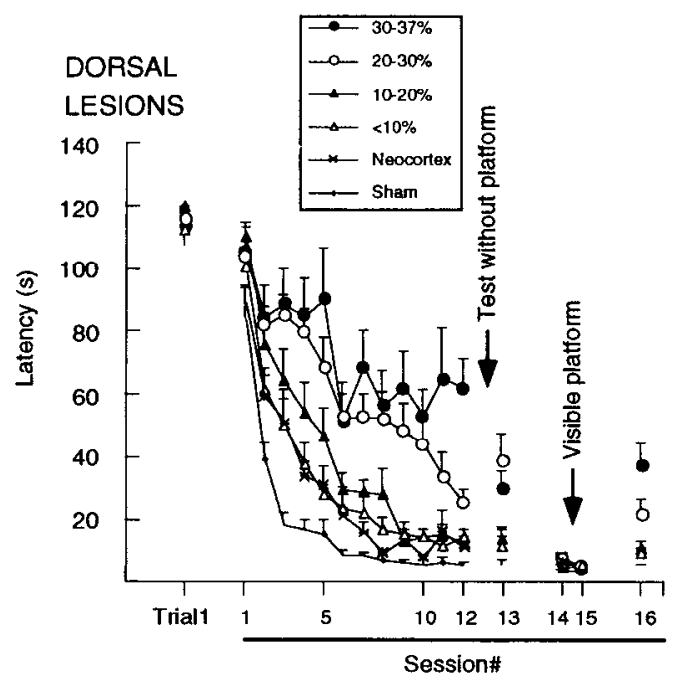

B

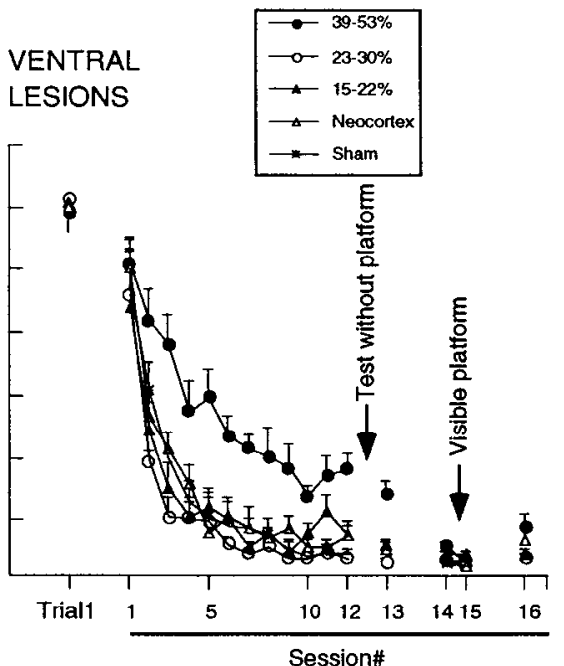

Figure 4. Differential effect on spatial learning of dorsal and ventral hippocampal lesions. The latency to locate the platform is plotted as a function of the size of the hippocampal lesions in rats with damage to the dorsal $(A)$ or the ventral $(B)$ hippocampus. The performances of animals with neocortical damage and sham-operated rats are also shown. The platform was submerged in sessions $1-13$ and 16 and was visible on sessions 14 and 15 . 


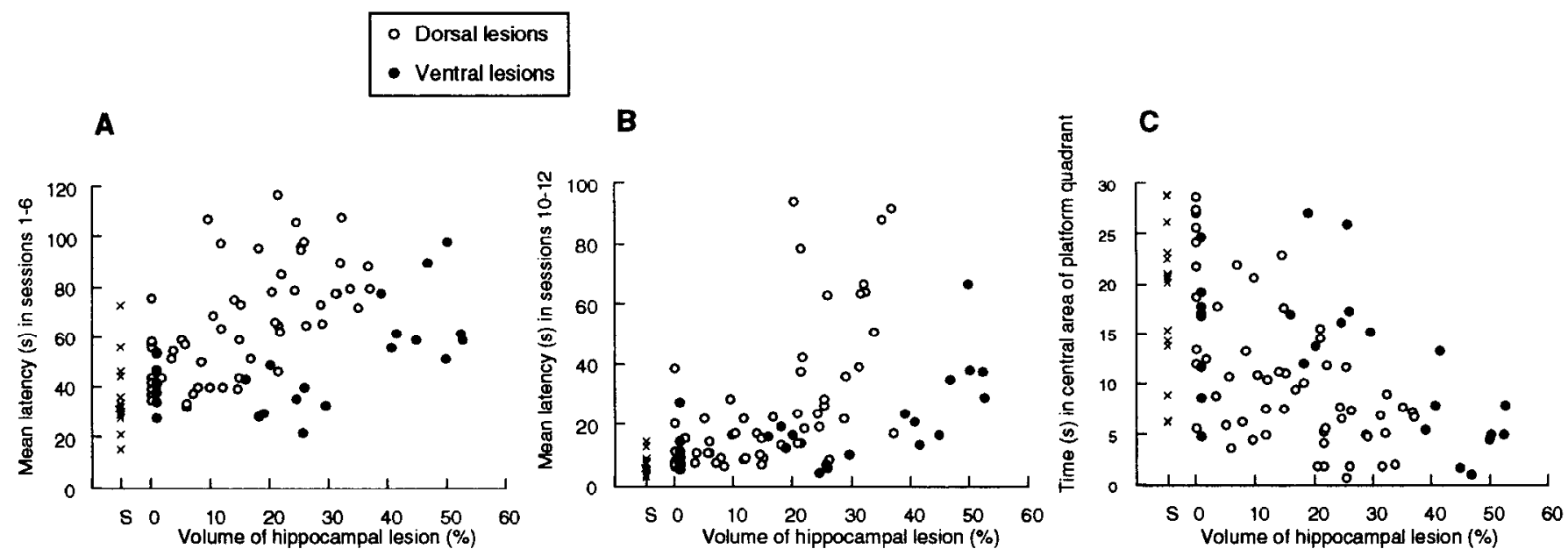

Figure 5. Relation between the size of the dorsal and ventral hippocampal lesions and learning performance. Scatter plots of the lesion volume against the mean latency in sessions $1-6(A)$, the mean latency in sessions $10-12(B)$, and the time swum inside a small circle $(52 \mathrm{~cm}$ diameter) around the previous platform position on the spatial probe test $(C)$. Each data point represents an individual rat. The performance of the shamoperated rats $(S)$ is shown for comparison. Open circles, dorsal hippocampal lesions; solid circles, ventral hippocampal lesions.

hippocampus, produced long-lasting impairment (escape latencies of $20-40 \mathrm{sec}$ ). In contrast, ventral hippocampal lesions had no effect on the latency to find the hidden platform unless they included almost the entire ventral half of the hippocampus.

Dorsal lesions. There was a nearly linear relation between the volume of the hippocampal lesion and the efficiency of learning (Fig. 5A): the larger the lesion, the longer the latency to find the hidden platform during the training [Spearman rank correlation between the hippocampal damage $(0-37 \%)$ and the mean latency on the first six sessions: $r=0.68, p<0.001]$. The latencies at which the behavior stabilized itself (mean of sessions 10-12) also depended on the hippocampal volume (Spearman rank correlation: $r=0.68, p<0.001$ ), but the relation was nonlinear (Fig. $5 B$ ): rats with damage to less than $18-20 \%$ escaped fast whereas those with more damage had longer latencies. Less pronounced correlations were seen between the neocortical lesion volumes and the latencies on sessions 1-6 $(r=0.40, p<$ $0.005)$ and sessions $10-12(r=0.32, p<0.01)$. ANOVA of the escape latencies (sessions 1-13) of the two control groups and four groups with hippocampal lesions (those with $0-10 \%, 10-$ $20 \%, 20-30 \%$, and 30-40\% damage) showed significant group $[F(5,60)=22.1, p<0.001]$ and group $\times \operatorname{session}[F(52,619)=$ $2.4, p<0.001]$ effects.

Ventral lesions. In the ventral groups, all rats with less than $30-40 \%$ total hippocampal damage escaped as fast as the shamoperated rats and the ventral neocortical control group, during both early and late stages of the training (Figs. 4, 5A,B). Although rats with more than $39 \%$ damage had longer escape latencies, they still escaped faster than the rats with the largest dorsal lesions. On the final session (16), the rats with the largest ventral lesions escaped nearly as fast as those with smaller ventral lesions or only neocortical damage (16 sec vs 6-12 sec). The hippocampal lesion volume correlated significantly but nonlincarly with the escapc latencics on sessions 1-6 (Spcarman $r=$ $0.56, p<0.005$; Fig. $5 A)$ and sessions 10-12 $(r=0.61, p<$ 0.005 ; Fig. $5 B$ ). There was also a weak correlation between these escape latencies and the amount of neocortical damage ( $r$ values $>0.30, p$ values $<0.06$ ). ANOVA of the escape latencies of the two control groups and rats with either small (15-30\%) or large (39-52\%) ventral hippocampal lesions showed significant group $[F(3,28)=16.1, p<0.001]$ and group $\times \operatorname{session}[F(32,299)$ $=1.45, p=0.05]$ effects.

We finally compared the performance of rats with roughly similar amounts of damage in the dorsal and the ventral hippocampus. All rats with damage exceeding $15 \%$ of the hippocampus (corresponding to the smallest ventral lesion) were evaluated. Four size categories were defined: 0\% (neocortical control), $15-22 \%, 23-30 \%$, and $>30 \%$. An ANOVA of the escape latencies during the training period revealed a highly significant effect of the location of the damage $[F(1,61)=34.0, p<0.001]$. The location effect depended on the size of the lesion [location $x$ size effect: $F(4,61)=8.7, p<0.001]$. The rats with ventral hippocampal damage were superior in all hippocampal lesion groups (15-22\% and 22-30\%: $p$ values $<0.001$; above $30 \%: p$ $<0.01)$. There was also a significant location $\times$ session effect $[F(10,597)=2.4, p<0.01]$, reflecting faster learning in the ventral groups. The dorsal and ventral neocortical groups, in contrast, did not differ $(p>0.10)$.

\section{Spatial probe test}

One way to estimate the spatial memory is to see whether trained animals search at the target location on a single trial when the platform is absent (Morris, 1984). We performed such a test and measured the time the rats spent inside a circle $(52 \mathrm{~cm}$ diameter) around the center of each quadrant (Fig. 6A). One of the center positions was identical to the previous platform location. We also got a precision measure, by recording the time spent inside the platform quadrant within three equally large areas defined by concentric circles $(52,74$, and $90 \mathrm{~cm}$ diameter) around the platform position (Fig. $6 D$ ) and the center of the three other quadrants.

Dorsal lesions. The size of the lesion in the dorsal hippocampus and the performance on the spatial probe test were negatively correlated (Fig. $5 \mathrm{C}$ ). The larger the lesion in the dorsal hippocampus, the less time the rats spent inside the inner circle (Spearman $r=-0.55, p<0.001)$ as well as the outer circle $(r$ $=-0.45, p<0.001)$ around the previous platform position. Whereas the sham-operated and the neocortical controls swam persistently in the central area of the platform quadrant (means of 18.0 and $19.1 \mathrm{sec}$, respectively), many of the rats with less 
A

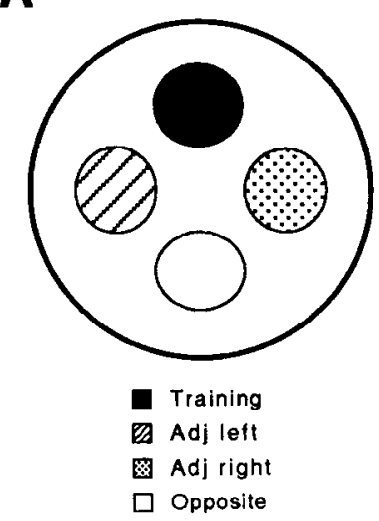

D

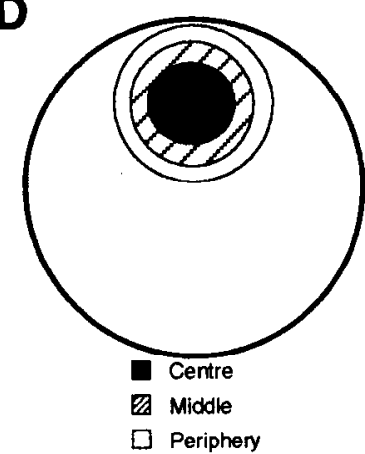

B

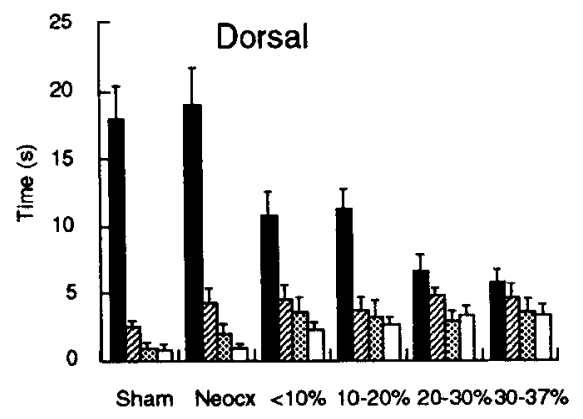

E

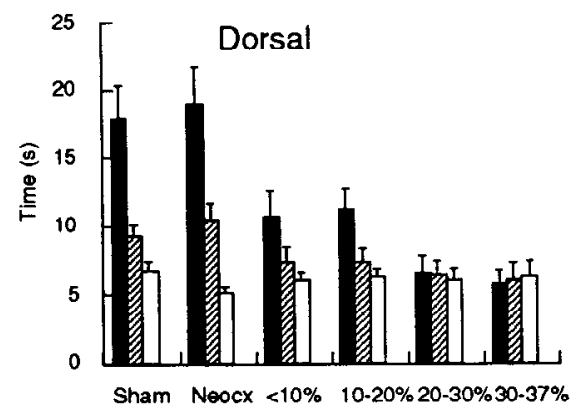

C
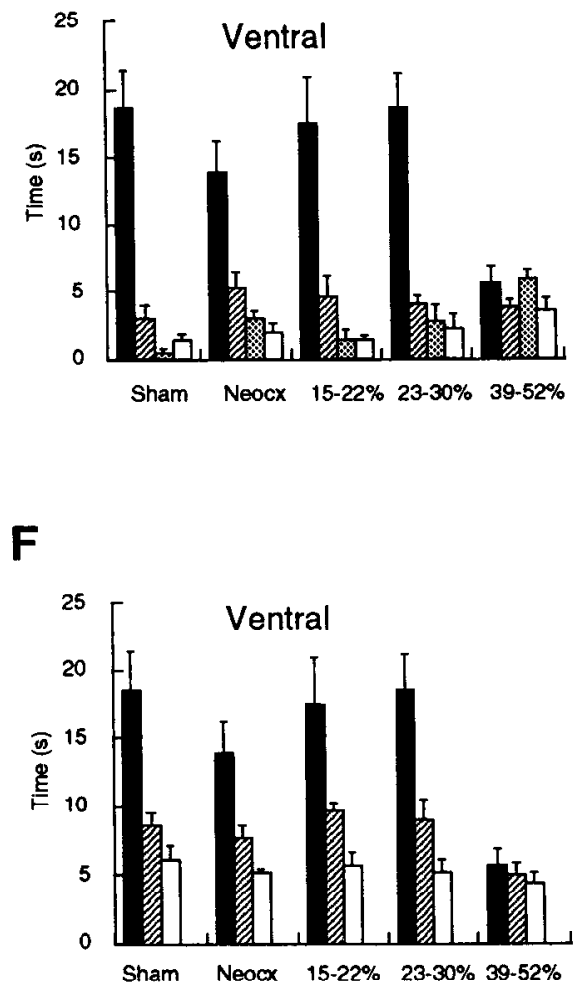

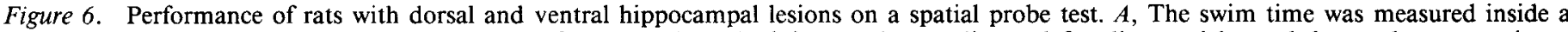

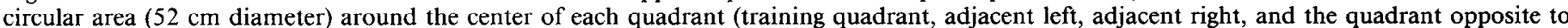

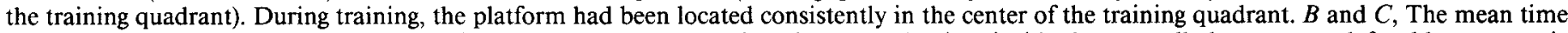

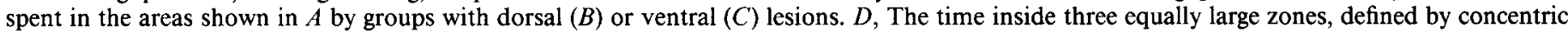

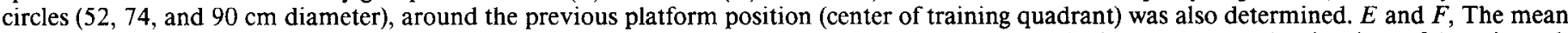

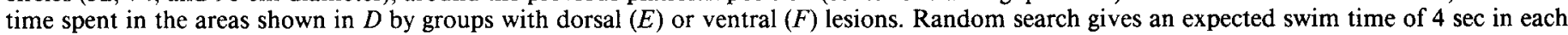
of the areas in $A$ and $D$.

than $20 \%$ hippocampal damage showed only a slight preference for this area (mean values: $10.8 \mathrm{sec}$ in the $<10 \%$ group, 11.3 sec in the 10-20\% group), but the search precision was quite variable within these groups. They did manage to direct their search toward the much larger area within the outer circle, however. Half of the rats with $20-30 \%$ and most of those with above $30 \%$ damage showed no spatial bias at all $(6.6 \mathrm{sec}$ in the central area in the $20-30 \%$ group, $5.8 \mathrm{sec}$ in the $>30 \%$ group). The neocortical lesion volume of the dorsal hippocampal and dorsal neocortical animals was not related to the time spent in any of the areas $(|r|$ values $<0.03, p$ values $>0.50)$. ANOVA of the time distribution of the two control and four hippocampal groups showcd significant group $\times$ quadrant $[F(12,140)=3.81, p<$ $0.001]$ and group $\times$ quadrant $\times$ circle $[F(14,169)=5.93, p<$ $0.001]$ effects.

Ventral lesions. All rats with ventral damage to less than $30 \%$ of the hippocampus spent just as much time in the vicinity of the previous platform position as the neocortical and shamoperated control animals (means of $18.1,13.9$, and $15.8 \mathrm{sec}$ in the central area, respectively; Figs. $5 C, 6 C, F$ ). Larger ventral lesions were associated with poorer performance (Fig. $5 C$ ). There was a significant, but moderate, relation between the size of the ventral lesions and the time inside the inner circle (Spearman $r=-0.47, p<0.01$; Fig. $6 C$ ) and the outer circle (Spearman $r=-0.43, p<0.025$ ) of the platform quadrant. Also the neocortical damage correlated with the time the rats swam inside the small circle $(r=-0.45, p=0.01)$ and the large circle $(r=$ $-0.55, p<0.005)$ of the platform quadrant. An ANOVA was performed on the time distribution of the two control groups and the two ventral hippocampal groups. The group $\times$ quadrant $[F(8,72)=6.27, p<0.001]$ and group $\times$ quadrant $\times$ circle $[F(6,59)=3.57, p<0.005]$ effects were significant.

An ANOVA of the performance of groups with $0 \%, 15-22 \%$, $23-30 \%$, or $>30 \%$ hippocampal damage confirmed that the rats with ventral lesions showed a clearer spatial bias toward the center of the platform quadrant than the dorsal ones [location $\times$ quadrant $\times$ circle effect: $F(3,168)=6.46, p<0.001$; location $\times$ quadrant effect was nonsignificant]. The analysis also confirmed that the dorsal-ventral difference depended on the size of the lesion [location $\times$ size $\times$ quadrant $\times$ circle: $F(11,168)=$ $2.74, p<0.0051$.

\section{Visible platform}

To control for any sensory or motor deficits of the impaired rats, we tested the rats in the standard visible-platform version of the water maze task (Morris, 1984). The task is nonspatial but the sensory, motor, and motivational requirements are similar to those of the spatial task. All groups of rats learned to locate the visible platform within a few seconds (Fig. 4). The lesion size showed no consistent relation to the performance ( $F$ values $<1, p$ values $>0.05)$. When the platform was submerged again in the subsequent session, the dorsal-ventral difference 
Figure 7. Spatial learning following dorsal CA1 lesions. $A$, Reconstruction of a representative dorsal CAI lesion. $B$, The latency to find the platform in rats with selective CAl lesions, neocortical control lesions, or sham operations. The platform was visible in session 16. The test without platform was conducted before session 15 . $C$, The mean time spent by the same groups inside a small ( $52 \mathrm{~cm}$ diameter) circle around the center of each quadrant (training quadrant, adjacent left, adjacent right, and the quadrant opposite to the training quadrant). During training, the platform had been located consistently in the center of the training quadrant. $D$, The mean time spent by the same groups inside equally large zones defined by three concentric circles $(52,74$, and $90 \mathrm{~cm}$ diameter) around the previous platform position (center of training quadrant).

\section{A}

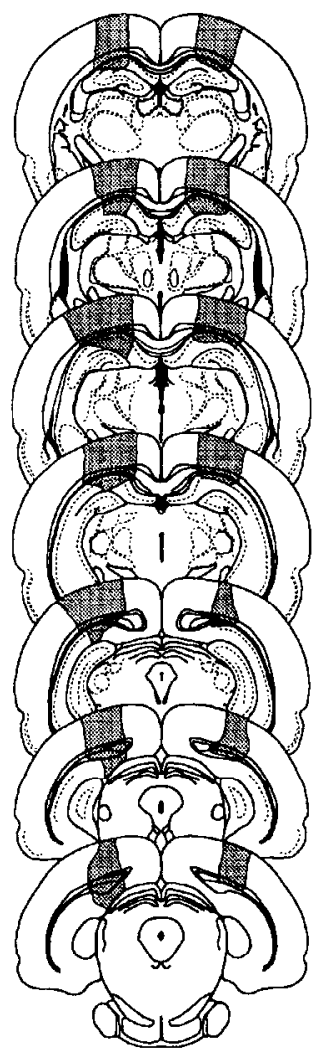

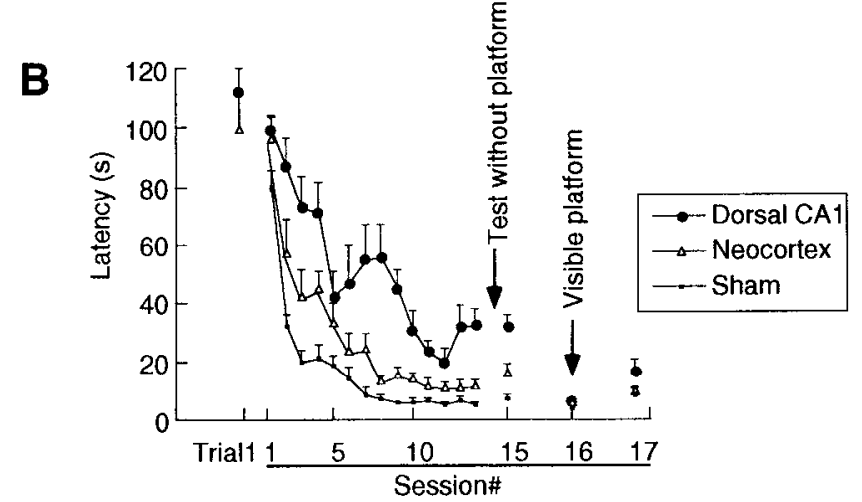
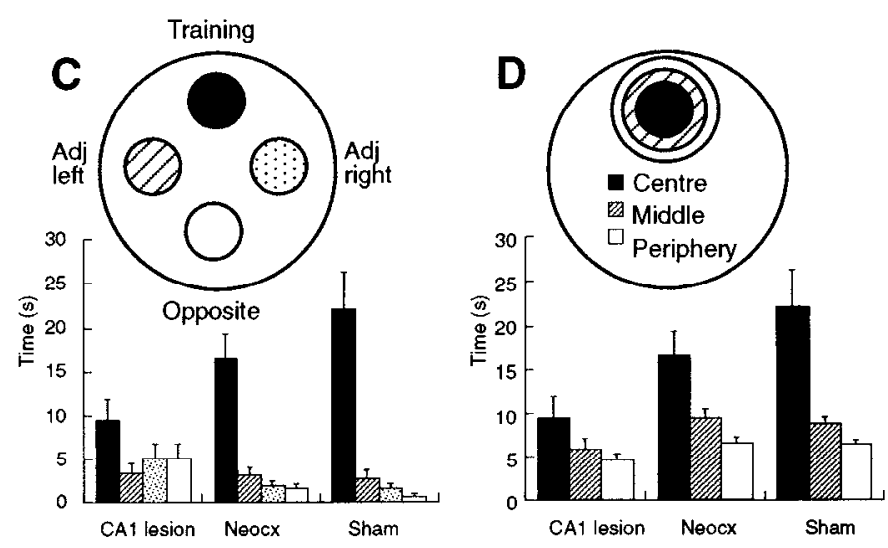

reappeared $(p<0.001)$. The complete absence of a lesion-related impairment in the cued task suggests that at least overt sensorimotor and motivational deficits cannot account for the deficits observed in the main experiment.

\section{Spatial learning following dorsal CAI lesions}

The pronounced effect of dorsal lesions could reflect transection of the fimbria and consequent subcortical denervation of the ventral hippocampus in the dorsal hippocampal groups. Thus, to be certain that no damage was made to the fimbria, we restricted some of the lesions to the CAl field.

Description of the lesions. The lesions $(n=7)$ were restricted to the septal one-third of the dorsal hippocampus (Fig. $7 \mathrm{~A}$ ) and affected from $15 \%$ to $30 \%$ of the entire CA1 volume (4-9\% of the total hippocampal tissue). The dentate gyrus was always exposed at the hippocampal fissure. Lesions invading the CA3 field were excluded. The neocortical lesions were larger than those of the previous experiments. The additional damage was mainly in the occipital cortex.

Performance in the water maze. The rats with CA1 lesions had longer escape latencies than neocortical and sham-operated control animals (Fig. 7B) and searched less persistently at the target location when the platform was absent (Fig. $7 C, D$ ). The learning impairment was just as large as the deficit produced by dorsal lesions of an equal proportion (15-30\%) of all cell fields (Figs. 4, 5). An ANOVA of the escape latencies (sessions 1-16) revealed a significant group effect $[F(2,10)=27.2, p<0.001]$, reflecting longer latencies in the $\mathrm{CA} 1$ damaged group than in the two control groups ( $p$ values $<0.005$ ). The group $\times$ session

interaction was not significant $(p>0.10)$. On the spatial probe test, weak group $\times$ quadrant $[F(5,49)=3.2, p<0.025]$ and group $\times$ quadrant $\times$ circle $[F(6,31)=2.4, p=0.05]$ effects were seen in the first half but not in the second half $(F$ values $<1)$ of the spatial probe test. Again, the effects reflected the difference between CAI and control animals ( $p$ values $<0.05$ ).

The effectiveness of the CA1 lesions, located far from the fimbria, suggests that any concomitant fimbrial damage is not the primary reason for the dorsal-ventral dissociation in the main experiments.

\section{Discussion}

The present study has three main results. First, we found that dorsal hippocampal lesions impair spatial learning more severely than equally large ventral lesions. In fact, the two ventral hippocampal halves had to be removed nearly totally to affect the acquisition of the task. Second, we observed a nearly linear relation between the volume of the dorsal hippocampal lesions and the learning of the spatial task. Third, we found a threshold value for the hippocampal lesion volume, below which spatial learning appeared relatively normal. The threshold was substantially lower for dorsal than for ventral lesions.

Spatial information processing takes place in the dorsal hippocampus. Our results suggest that the dorsal but not the ventral half of the hippocampus is sufficient to sustain spatial learning in the water maze. This view is based upon the virtual absence of spatial learning deficits after quite large ventral hippocampal lesions, while even small dorsal lesions gave deficits in the acquisition phase and in the spatial probe test. The rats with the 
larger dorsal lesions showed a lack of spatial preference on the spatial probe test comparable to that reported for rats with complete hippocampal aspiration lesions (Morris et al., 1982). The spatial probe test performance at the end of training is probably quite resistant against any small variation of the procedures used in the two laboratories. Further support for the importance of the dorsal hippocampus in spatial learning comes from studies in which radial-maze learning (Handelmann and Olton, 1981), acquisition of a learning-set task in a water maze (Auer et al., 1989), and spatial learning during exploration (Thinus-Blanc et al., 1991) were impaired by neurotoxic or ischemic damage, or lidocaine-induced blockade, restricted to the dorsal hippocampus. Admittedly, the latter studies did not test the effect of ventral lesions. However, Hughes (1965) and Sinnamon et al. (1978) observed larger deficits in two other types of maze learning following dorsal than ventral hippocampal lesions, although the size of these lesions was not quantified and the fimbria was often damaged by the dorsal lesions.

It may be argued that the efficiency of the dorsal hippocampal lesions could reflect inadvertent lesion of the fimbria, dissociating the ventral half from the rest of the hippocampus, or disruption of afferent aminergic or cholinergic fibers. Inadvertent fimbria lesions appear unlikely, since any such lesion, which must have been too small to be seen on the histological sections, is likely to affect fibers at the base of the fimbria, while the efferents from the ventral hippocampus course near the edge (Andersen et al., 1973; Swanson and Cowan, 1977). Second, dorsal CAl lesions, located far from the fimbria (Fig. $7 A$ ), impaired spatial learning as much as comparable lesions affecting all cell fields.

The role of damage to the efferent axons of the subiculum is more difficult to evaluate. Since some of these fibers pass on the alvear surface of the dorsal CAl (Swanson and Cowan, 1977), it is likely that dorsal lesions may cut more of these fibers than ventral hippocampal lesions do. Some of the subiculofugal fibers may be involved in spatial learning, as damage to the fornix-fimbria at a septal level impairs the acquisition of a water maze task (Sutherland and Rodriguez, 1989). On the other hand, in the present study, the most extensive damage to the subiculum and possibly also to the entorhinal cortex occurred in the ventral groups. The largest ventral lesions thus may have deprived the dorsal part of the dentate gyrus of some of its perforant path input, resulting in a deficit that was actually due to partial deafferentation of the dorsal hippocampus.

The dorsal lesion may also have damaged aminergic or cholinergic fibers destined for the ventral hippocampus. For example, lesions of serotoninergic fibers, which enter through a dorsal route (Storm-Mathiesen and Guldberg, 1974), impair learning when combined with damage to cholinergic afferents (Nilsson et al., 1988). Such a combined effect may have given an additive effect to the dorsal lesion in the present study. Deafferentation of noradrenergic fibers to the hippocampus is unlikely to contribute to the dorsoventral difference in spatial learning since most of these axons enter the hippocampus ventrally (Storm-Mathiesen and Guldberg, 1974). In conclusion, a proportion of the effects of the dorsal and ventral lesions may reflect partial deafferentation of the remaining hippocampus. These questions may be answered by future experiments with cytotoxic lesions.

Effect of lesion volume. A correlation between the amount of hippocampal damage and performance was observed in rats learning spatial alternation (Volpe et al., 1992) as well as in the present study. The correlation between the volume of the dorsal lesion and spatial learning could reflect the involvement of an extended neuronal network, in which smaller elements are responsible for parts of the function, but the whole network for a complete performance. Alternatively, a specific part of the network could serve as a crucial substrate, being damaged only by the more extensive lesions. The increasing learning deficit with larger, concentrically arranged lesions (Fig. 1) suggests that activity of a large part of the dorsal hippocampus is required for full spatial learning abilities, thus supporting the network idea. In other words, spatial learning requires cooperation between several of the postulated lamellae (Andersen et al., 1971).

Our findings may also be related to recent neuroethological evidence, which suggests that spatial learning ability may depend on the amount of available hippocampal tissue (Sherry et al., 1992). Relative to the body mass, the hippocampus is larger in food-storing birds, for which spatial memory is important, than in related nonstoring species (Krebs et al., 1989; Sherry et al., 1989; Healy and Krebs, 1992). Polygamous male voles, which have larger range sizes than females of their own species and both sexes of a related monogamous species (Gaulin and FitzGerald, 1988), also have larger hippocampi than these other animals (Jacobs et al., 1990). Thus, selection for spatial learning abilities seems to concur with selection for hippocampal size. Taken together with our results, these bird and vole data suggest that spatial learning is dependent on the recruitment of a minimum number of cells and neural circuits distributed in a large part of the hippocampus.

A volume threshold for efficient lesions. Our results suggest that a minimal lesion volume of the dorsal hippocampus is needed to impair spatial learning. A threshold value of about $20 \%$ damage was required to produce a lasting deficit in escape latency. Since the rate of acquisition and the performance on the spatial probe test were reduced by even smaller lesions, the threshold for inducing a spatial learning and retention deficit may be even lower. Our threshold estimate is close to that of a recent study in monkeys, in which ischemia-induced damage to $24 \%$ of the CA1 field produced a delayed-nonmatching-to-sample memory impairment as severe as the deficit caused by a complete hippocampal lesion (Zola-Morgan et al., 1992). In contrast, in pretrained rats with ischemic or ibotenic acid-induced hippocampal lesions, as much as two-thirds of the dorsal hippocampus had to be damaged in order to impair the acquisition of a spatial alternation task (Volpe et al., 1992). The different threshold from that of the present study may be explained if spatial alternation in pretrained rats is a less sensitive measure of hippocampal function than the water maze.

Dorsal and ventral hippocampus may have different functions. Although the synaptic organization is similar along the entire septotemporal axis of the hippocampus (Andersen et al., 1971), the dorsal and ventral parts process different kinds of information. A functional dissociation may be related to the different connectivity of the dorsal and the ventral hippocampus. Sensory information from several cortical association areas and from the olfactory bulb reaches mainly the dorsal half of the hippocampus by way of the lateral entorhinal area (Jones and Powell, 1970; van Hoesen and Pandya, 1975; Deacon et al., 1983; Room and Groenewegen, 1986; Insausti et al., 1987). Olfactory fibers also reach the lateral entorhinal area-and thence the dorsal half of the hippocampus. In addition, some olfactory axons terminate in the anteromedial entorhinal and the neighboring periamygdaloid cortices (Beckstead, 1978; Kosel et al., 1981). From 
these regions, cells project to the ventral hippocampus. Thus, the ventral hippocampus receives olfactory information in addition to signals from the amygdala, the hypothalamus, and the medial septal and basal forebrain nuclei (Beckstead, 1978). With such a pattern of organization, the relevant sensory information during performance in a water maze would be processed mainly in the dorsal part of the hippocampus, a fact that is consistent with our results. Conversely, with different afferent information, the ventral hippocampus may have a different physiological role than its dorsal counterpart, possibly relatcd to cmotional or hormonal responses or their integration with olfactory signals.

Our data do not allow a prediction of the functional role of the ventral hippocampus. The dorsal and ventral parts of the hippocampus may process qualitatively different kinds of information. Alternatively, the two parts may do the same task, the dorsal half just being more efficient. However, the distribution of the escape latency in the water maze for animals with dorsal and ventral lesions strongly suggests that these lesions produce qualitatively different results, and not only various degrees of the same deficit. To be effective, the ventral lesions had to be so large as to approach the dorsal hippocampus, and may, in fact, have lesioned some cells in the latter.

\section{References}

Amaral DG, Witter MP (1989) The three-dimensional organization of the hippocampal formation: a review of anatomical data. Neuroscience 31:571-591.

Amaral DG, Witter MP, eds (1991) Hippocampus forum: is the hippocampal formation preferentially involved in spatial behavior? Hippocampus 1:221-292.

Andersen P, Bliss TVP, Skrede KK (1971) Lamellar organization of hippocampal excitatory pathways. Exp Brain Res 13:222-238.

Andersen P, Bland BH, Dudar JD (1973) Organization of hippocampal output. Exp Brain Res 17:152-168.

Auer RN, Jensen ML, Whishaw IQ (1989) Neurobehavioral deficit due to ischemic damage limited to half of the CAl sector of the hippocampus. J Neurosci 9:1641-1647.

Beckstead RM (1978) Afferent connections of the entorhinal area in the rat as demonstrated by retrograde cell-labeling with horseradish peroxidase. Brain Res 152:249-264.

Deacon TW, Eichenbaum H, Rosenberg P, Eckmann KW (1983) Afferent connections of the perirhinal cortex in the rat. J Comp Neurol 220:168-190.

Eichenbaum H, Fagan A, Mathews P, Cohen NJ (1988) Hippocampal system dysfunction and odor discrimination learning in rats: impairment or facilitation depending on representational demands. Behav Neurosci 102:331-339.

Eichenbaum H, Mathews P, Cohen NJ (1989) Further studies of hippocampal representation during odor discrimination learning. Behav Neurosci 103:1207-1216.

Gaulin SJC, FitzGerald RW (1988) Home-range size as a predictor of mating systems in Microtus. J Mammal 69:311-319.

Gross CG, Chorover SL, Cohen SM (1965) Caudate, cortical, hippocampal and dorsal thalamic lesions in rats: alternation and HebbWilliams maze performance. Neuropsychologia 3:53-68.

Handelmann GE, Olton DS (1981) Spatial memory following damage to hippocampal CA3 pyramidal cells with kainic acid: impairment and recovery with preoperative training. Brain Res 217:41-58.

Healy SD, Krebs JR (1992) Food storing and the hippocampus in corvids: amount and volume are correlated. Proc R Soc Lond [Biol] 248:241-245.

Hughes KR (1965) Dorsal and ventral hippocampus lesions and maze learning: influence of preoperative environment. Can J Psychol 19: 325-332.

Huynh H, Feldt LS (1976) Estimation of the Box correction for degrees of freedom from sample data in the randomized block and split-plot designs. J Educ Stat 1:69-82.

Insausti R, Amaral DG, Cowan WM (1987) The entorhinal cortex of the monkey. II. Cortical afferents. J Comp Neurol 264:356-395.
Jacobs LF, Gaulin SJC, Sherry DF, Hoffman GE (1990) Evolution of spatial cognition: sex-specific patterns of spatial behavior predict hippocampal size. Proc Natl Acad Sci USA 87:6349-6352.

Jarrard LE (1978) Selective hippocampal lesions: differential effects on performance by rats of a spatial task with preoperative versus postoperative training. J Comp Physiol Psychol 92:1119-1127.

Jones EG, Powell TPS (1970) An anatomical study of converging sensory pathways within the cerebral cortex of the monkey. Brain 93: 793-820.

König JFR, Klippel RA (1963) The rat brain: a stereotaxic atlas of the forebrain and lower parts of the brain stem. Baltimore: Williams and Wilkins.

Kosel KC, van Hoesen GW, West JR (1981) Olfactory bulb projections to the parahippocampal area of the rat. J Comp Neurol 198:467-482.

Krebs JR, Sherry DF, Healy SD, Perry VH, Vaccarino AL (1989) Hippocampal specialization of food-storing birds. Proc Natl Acad Sci USA 86:1388-1392.

Morris RGM (1984) Developments of a water-maze procedure for studying spatial learning in the rat. J Neurosci Methods 11:47-60.

Morris RGM, Garrud P, Rawlins JNP, O'Keefe J (1982) Place navigation impaired in rats with hippocampal lesions. Nature 297:681683.

Morris RGM, Schenk F, Tweedie F, Jarrard LE (1990) Ibotenate lesions of hippocampus and/or subiculum: dissociating components of allocentric spatial learning. Eur $\mathbf{J}$ Neurosci 2:1016-1028.

Muller RU, Kubie JL, Ranck JB Jr (1987) Spatial firing patterns of hippocampal complex-spike cells in a fixed environment. J Neurosci 7:1935-1950.

Nadel L (1968) Dorsal and ventral hippocampal lesions and behavior. Physiol Behav 3:891-900.

Nilsson OG, Strecker RE, Daszuta A, Björklund A (1988) Combined cholinergic and serotonergic denervation of the forebrain produces severe deficits in a spatial learning task in the rat. Brain Res 453:235246 .

O'Keefe J, Conway DH (1978) Hippocampal place units in the freelymoving rat: why they fire when they fire. Exp Brain Res 31:573-590.

O'Keefe J, Dostrovsky J (1971) The hippocampus as a spatial map. Preliminary evidence from unit activity in the freely-moving rat. Brain Res 34:171-175.

Olton DS, Walker JA, Gage FH (1978) IIippocampal connections and spatial discrimination. Brain Res 139:295-308.

Quirk GJ, Muller RV, Kubie JL (1990) The firing of hippocampal place cells in the dark depends on the rat's recent experience. J Neurosci 10:2008-2017.

Room P, Groenewegen HJ (1986) Connections of the parahippocampal cortex. I. Cortical afferents. J Comp Neurol 251:415-450.

Rudy JW, Sutherland RJ (1989) The hippocampal formation is necessary for rats to learn and remember configural discriminations. Behav Brain Res 34:97-107.

Ruth RE, Collier TJ, Routtenberg A (1982) Topography between the entorhinal cortex and the dentate septotemporal axis in rats. I. Medial and intermediate entorhinal projecting cells. J Comp Neurol 209:6978.

Sherry DF, Vaccarino AL, Buckenham K, Herz RS (1989) The hippocampal complex of food-storing birds. Brain Behav Evol 34:308317.

Sherry DF, Jacobs LF, Gaulin SJC (1992) Spatial memory and adaptive specialization of the hippocampus. Trends Neurosci 15:298-303.

Sinnamon HM, Freniere S, Kootz J (1978) Rat hippocampus and memory for places of changing significance. J Comp Physiol Psychol 92:142-155.

Stevens R, Cowey A (1973) Effects of dorsal and ventral hippocampal lesions on spontaneous alternation, learned alternation and probability learning in rats. Brain Res 52:203-224.

Storm-Mathiesen J, Guldberg HC (1974) 5-Hydroxytryptamine and noradrenaline in the hippocampal region: effect of transection of afferent pathways on endogenous levels, high affinity uptake and some transmitter-related enzymes. J Neurochem 22:793-803.

Sutherland RJ, Rodriguez AJ (1989) The role of the fornix/fimbria and some related subcortical structures in place learning and memory. Behav Brain Res 32:265-277.

Sutherland RJ, Whishaw IQ, Kolb B (1983) A behavioural analysis of spatial localization following electrolytic, kainate or colchicineinduced damage to the hippocampal formation in the rat. Behav Brain Res 7:133-153. 
Sutherland RJ, McDonald RJ, Hill CR, Rudy JW (1989) Damage to the hippocampal formation in rats selectively impairs the ability to learn cue relationships. Behav Neural Biol 52:331-356.

Swanson LW, Cowan WM (1977) An autoradiographic study of the organization of the efferent connections of the hippocampal formation in the rat. $J$ Comp Neurol 172:49-84.

Thinus-Blanc C, Save E, Poucet B, Buhot MC (1991) The effects of reversible inactivations of the hippocampus on exploratory activity and spatial memory. Hippocampus 1:365-372.

van Hoesen GW, Pandya DN (1975) Some connections of the entorhinal (area 28) and perirhinal (area 35) cortices of the rhesus monkey. I. Temporal lobe afferents. Brain Res 95:1-24.

Vasey MW, Thayer JF (1987) The continuing problem of false positives in repeated measures ANOVA in psychophysiology: a multivariate solution. Psychophysiology 24:479-486.
Volpe BT, Davis HP, Towle A, Dunlap WP (1992) Loss of hippocampal CAl pyramidal neurons correlates with memory impairment in rats with ischemic or neurotoxin lesions. Behav Neurosci 106:457464 .

Witter MP, Groenewegen HJ (1984) Laminar origin and septotemporal distribution of entorhinal and perirhinal projections to the hippocampus in the cat. J Comp Neurol 224:371-385.

Witter MP, van Hoesen GW, Amaral DG (1989) Topographical organization of the entorhinal projection to the dentate gyrus of the monkey. J Neurosci 9:216-228.

Zilles K (1985) The cortex of the rat. A stereotaxic atlas. Berlin: Spring-

Zola-Morgan S, Squire LR, Rempel NL, Clower RP, Amaral DG (1992) Enduring memory impairment in monkeys after ischemic damage to the hippocampus. J Neurosci 12:2582-2596. 